\title{
TILTING MODULES AND A THEOREM OF HOSHINO
}

\section{by IBRAHIM ASSEM and OTTO KERNER}

(Received 29 July, 1990)

Introduction. Let $k$ be an algebraically closed field, and $A$ a finite dimensional $k$-algebra, which we shall assume, without loss of generality, to be basic and connected. By module is meant throughout a finitely generated right $A$-module. Following Happel and Ringel [10], we shall say that a module $T_{A}$ is a tilting (respectively, cotilting) module if it satisfies the following three conditions:

(1) $\operatorname{Ext}_{A}^{2}(T,-)=0$ (respectively, $\left.\operatorname{Ext}_{A}^{2}(-, T)=0\right)$;

(2) $\operatorname{Ext}_{A}^{1}(T, T)=0$;

(3) the number of non-isomorphic indecomposable summands of $T$ equals the rank of the Grothendieck group $K_{0}(A)$ of $A$.

These modules have found, since their introduction, numerous applications in the representation theory of algebras (see [2, 9]); hence the interest in constructing them. One method of construction uses torsion theories. Given a tilting (respectively, cotilting) module $T_{A}$, the full subcategories

and

$$
\begin{aligned}
& \mathscr{T}_{*}(T)=\left\{M_{A} \mid \operatorname{Ext}_{A}^{1}(T, M)=0\right\} \\
& \mathscr{F}_{*}(T)=\left\{M_{A} \mid \operatorname{Hom}_{A}(T, M)=0\right\} \\
& \mathscr{T}^{*}(T)=\left\{M_{A} \mid \operatorname{Hom}_{A}(M, T)=0\right\} \\
& \left.\mathscr{F}^{*}(T)=\left\{M_{A} \mid \operatorname{Ext}_{A}^{1}(M, T)=0\right\}\right)
\end{aligned}
$$

respectively,

and

of the category $\bmod A$ of $A$-modules are respectively the torsion class and the torsion-free class of a torsion theory. Such a torsion theory is said to be a tilting (respectively, cotilting) torsion theory. Conversely, Hoshino has shown in [12] that, if $(\mathscr{T}, \mathscr{F})$ is a torsion theory on $\bmod A$ such that $\mathscr{T}$ contains all injective modules, and $\mathscr{T}$ or $\mathscr{F}$ contains only finitely many non-isomorphic indecomposable modules (we then say, by abuse of language, that $\mathscr{T}$ or $\mathscr{F}$ is finite), then $(\mathscr{T}, \mathscr{F})$ is a tilting torsion theory (see also [1], [18]). While the first condition is obviously necessary, the second is not as is shown by the following example. Let $A$ be a wild hereditary algebra with at least three non-isomorphic simple modules; it was proved by Ringel ([16], see also [6]) that $A$ has a regular tilting (and cotilting) module. For such a module, all the indecomposable preprojective modules belong to the torsion-free class, while all the indecomposable preinjective modules belong to the torsion class, so that both are infinite.

In this paper, we consider classes of algebras having the property that, for every tilting or cotilting torsion theory $(\mathscr{T}, \mathscr{F}), \mathscr{T}$ or $\mathscr{F}$ is finite. This is quite useful in practice, since in many applications, it is easier to start by constructing the torsion theory, then finding the corresponding module (this method was heavily applied, for instance, in [9, $(I V, 7)])$. Since all representation-finite algebras obviously satisfy this property, we shall be mainly interested in representation-infinite algebras. It was shown in $[\mathbf{1 1},(3)]$ that the tame hereditary algebras satisfy the stated property. We shall prove the following generalisation of this result.

THEOREM A. Let $A$ be an iterated tilted algebra of euclidean type, and $T_{A}$ a tilting (respectively, cotilting) module. Then $\mathscr{T}_{*}(T)$ or $\mathscr{F}_{*}(T)$ (respectively, $\mathscr{T}^{*}(T)$ or $\mathscr{F}^{*}(T)$ ) is finite. 
On the other hand, this property is not satisfied by the tubular algebras of [15] (because of the existence of regular tilting modules). It follows directly from the theorem that the stated property is satisfied by the tilted algebras of euclidean type. It is well-known that such an algebra is either representation-finite or one-parametric (in the sense of $[17,(2.1)])$. Also, this property is easily seen to be satisfied by a hereditary algebra with two non-isomorphic simple modules. Our second theorem shows that, up to finite enlargements, these are the only classes of tilted algebras which satisfy this property.

THEOREM B. Let $A$ be a tilted algebra. The following conditions are equivalent:

(1) for every tilting module $T_{A}, \mathscr{T}_{*}(T)$ or $\mathscr{F}_{*}(T)$ is finite, and for every cotilting module $T_{A}^{\prime}, \mathscr{T}^{*}\left(T^{\prime}\right)$ or $\mathscr{F}^{*}\left(T^{\prime}\right)$ is finite;

(2) if $A$ is tame, then $A$ is representation-finite or one-parametric and, if $A$ is wild, then one of the end algebras of $A$ is zero, and the other is hereditary with two non-isomorphic simple modules.

Clearly, if $T_{A}$ is a regular tilting module, the equivalent conditions of the theorem are not satisfied, but the converse is not true, as will be shown in the course of the proof. The paper is organised as follows. In Section 1, we quote the definitions and results that will be used in the sequel, prove Theorem A, then consider the case of the tubular algebras. Section 2 is devoted to the proof of Theorem B.

\section{Iterated tilted algebras of euclidean type.}

1.1. Let $A$ be a finite dimensional algebra. It is well-known that for any (basic) algebra $A$, there exists a bound quiver $(Q, I)$ such that we have an isomorphism $A \cong k Q / I$. A bound quiver algebra $A=k Q / I$ can equivalently be considered as a locally bounded $k$-linear category with object class the set of points in $Q$, and set of morphisms from $x$ to $y$ the vector space $k Q(x, y)$ of all linear combinations of paths in $Q$ from $x$ to $y$ modulo the subspace $I(x, y)=I \cap k Q(x, y)$, see [8]. A full subcategory $C$ of $A$ is called convex if any path in $A$ with source and target in $C$ lies entirely in $C$. We shall use freely and without further reference properties of the category $\bmod A$, the Auslander-Reiten translations $\tau=D \operatorname{Tr}$ and $\tau^{-1}=\operatorname{Tr} D$, and the Auslander-Reiten quiver $\Gamma_{A}$ of $A$, as can be found, for instance in [5], [15]. Recall that a component of $\Gamma_{A}$ is called preprojective (respectively, preinjective) if it contains no oriented cycle, and each indecomposable module in this component is of the form $\tau^{-t} P$, with $t \geq 0$ and $P$ projective (respectively, $\tau I$, with $s \geq 0$ and $I$ injective). We shall denote by $P(a)$ (respectively, $I(a)$ ) the indecomposable projective (respectively, injective) module corresponding to a point $a$ in the ordinary quiver of $A$. The support of a module $M_{A}$ is the full subcategory of $A$ consisting of all objects $a$ such that $\operatorname{Hom}_{A}(P(a), M) \neq 0$.

For tilting theory and iterated tilted algebras, we refer to [2], [10]. By the Brenner-Butler theorem, if $A$ is an algebra, $T_{A}$ a tilting module and $B=$ End $T_{A}$, then the functors $\operatorname{Hom}_{A}(T,-)$ and $-\bigotimes_{B} T$ induce mutually inverse equivalences between the full subcategories $\mathscr{T}_{*}(T)$ of $\bmod A$ and $\mathscr{Y}_{*}(T)=\left\{N_{B} \mid \operatorname{Tor}_{1}^{B}(N, T)=0\right\}$ of $\bmod B$, while the functors $\operatorname{Ext}_{A}^{1}(T,-)$ and $\operatorname{Tor}_{1}^{B}(-, T)$ induce mutually inverse equivalences between the full subcategories $\mathscr{F}_{*}(T)$ of $\bmod A$ and $\mathscr{X}_{*}(T)=\left\{N_{B} \mid N \otimes_{B} T=0\right\}$ of $\bmod B$. Further, $\left(\mathscr{X}_{*}(T), \mathscr{Y}_{*}(T)\right)$ is a torsion theory on $\bmod B$, which is splitting if $A$ is hereditary. Similar results hold for cotilting modules. 
If $\Delta$ is a finite connected quiver without oriented cycles, an algebra $A$ is called iterated tilted of type $\Delta$ if there exist a sequence of algebras $A=A_{0}, A_{1}, \ldots, A_{m}=k \Delta$ and a sequence $T_{A_{i}}^{(i)}(0 \leq i<m)$ of tilting or cotilting modules such that $A_{i+1}=\operatorname{End} T_{A_{i}}^{(i)}$ for each $i$ (for equivalent definitions, see [2], [9]). If $\Delta$ is a Dynkin (respectively, euclidean, wild) quiver, then $A$ is said to be of Dynkin (respectively, euclidean, wild) type. If $m \leq 1$, then $A$ is called tilted of type $\Delta$. Tilted algebras are characterised by the existence of complete slices $[15,(4.2)(3)]$. The structure of the representation-infinite iterated tilted algebras of euclidean type is described in [3, (2)]. In particular, each such algebra contains a unique full convex subcategory which is tame concealed (that is, which is the endomorphism algebra of a preprojective tilting module over a tame hereditary algebra). Further, the Auslander-Reiten quiver of such an algebra always has a unique preprojective and a unique preinjective component. All other components are called regular.

1.2. Lemma. Let $A$ be a representation-infinite iterated tilted algebra of euclidean type, and $T_{A}$ a tilting module without preprojective (respectively, preinjective) direct summands. Then all the homogeneous tubes in $\Gamma_{A}$ belong to $\mathscr{F}_{*}(T)$ (respectively, $\left.\mathscr{T}_{*}(T)\right)$.

Proof. If $T_{A}$ has no preprojective summands, its summands are regular or preinjective, but certainly not homogeneous (because indecomposable homogeneous modules have self-extensions). Thus it follows from the orthogonality of the tubes that, for every indecomposable homogeneous module $M$, we have $\operatorname{Hom}_{A}(T, M)=0$. The dual statement follows from the fact that, if all summands of $T$ are regular or proprojective, then, by the Auslander-Reiten formula, $\operatorname{Ext}_{A}^{1}(T, M)=D \operatorname{Hom}_{A}(M, \tau T)=0$ for every indecomposable homogeneous module $M_{A}$.

1.3. Corollary. Let $A$ be a representation-infinite iterated tilted algebra of euclidean type. Then $A$ has no regular tilting module and no regular cotilting module.

Proof. By (1.2), $A$ has no regular tilting module. That it has no regular cotilting module follows from the fact that by $[4,(2.5)]$ or $[9,(I V, 5.6)]$, its opposite algebra $A^{\text {op }}$ is also a representation-infinite iterated tilted algebra of euclidean type.

1.4. Proof of Theorem $A$. We may certainly assume that $A$ is representation-infinite. By duality, it suffices to prove the statement in case $T_{A}$ is a tilting module.

We first claim that, if $\mathscr{F}_{*}(T)$ is infinite, then infinitely many simple homogeneous $A$-modules belong to $\mathscr{F}_{*}(T)$.

This claim will be shown by induction on the rank $n$ of $K_{0}(A)$. If $n=2$, then $A$ is the Kronecker algebra (that is, is tame hereditary of type $\tilde{A}_{1,1}$ ) and the statement is trivial. Assume that the rank of $K_{0}(A)$ equals $n$. If $T_{A}$ has no preprojective direct summand, the statement follows from (1.2). Otherwise, observe that the preprojective direct summands of $T$ are partially ordered by the successor relation. We claim that $T$ may be assumed to have as direct summand a simple projective module.

Indeed, if this is not the case, let $P(a)$ be a simple projective module, and

$$
T[a]=\tau^{-1} P(a) \oplus\left(\bigoplus_{b \neq a} P(b)\right)
$$


the corresponding APR-tilting module [2, (1.6)]). Clearly, all direct summands of $T$ belong to $\mathscr{T}_{*}(T[a])$, and hence $\operatorname{Hom}_{A}(T[a], T)$ is a tilting $B$-module, where $B=$ End $T[a]$. On the other hand, the functor

$$
-\otimes_{B} T[a]: \bmod B \rightarrow \bmod A
$$

induces an equivalence $\mathscr{Y}_{*}(T[a]) \widetilde{T_{*}}(T[a])$ and its restriction to a functor $\mathscr{F}_{*}\left(\operatorname{Hom}_{A}(T[a], T)\right) \rightarrow \mathscr{F}_{*}(T)$ defines a full exact embedding of the category $\mathscr{F}_{*}\left(\operatorname{Hom}_{A}(T[a], T)\right)$ as a cofinite subcategory of $\mathscr{F}_{*}(T)$, which is closed under extensions. Furthermore, the functor

$$
\operatorname{Hom}_{A}(T[a],-): \bmod A \rightarrow \bmod B
$$

induces an equivalence between the full subcategories consisting of the homogeneous modules in $\bmod A$ and $\bmod B$, respectively. Inductively, applying a sequence of APR-tilts corresponding to simple projectives in the preprojective component, we reach a situation where $T$ has as direct summand a simple projective module. As we have seen, in each APR-tilting step, the torsion-free part induced by the image of $T$ differs by at most one indecomposable from the original one.

Assume thus that $T_{A}$ has as direct summand the simple projective $e A$, and let $A^{\prime}=A / A e A$. Then $A^{\prime}$ is a full convex subcategory of $A$ and consequently, by $[4,(5.2)]$, each of its connected components is iterated tilted of Dynkin or euclidean type. Also, by [14, (3.2)], all the summands of $T$ except $e A$ define a tilting $A^{\prime}$-module $T^{\prime}=T / T e A$ such that the canonical embedding $\bmod A^{\prime} \leftrightarrow \bmod A$ induces an identification $\mathscr{F}_{*}\left(T^{\prime}\right)=\mathscr{F}_{*}(T)$. In particular, $\mathscr{F}_{*}\left(T^{\prime}\right)$ is infinite and consequently at least one of the connected components of $A^{\prime}$ is a representation-infinite iterated tilted algebra of euclidean type. We may thus assume that $A^{\prime}$ is connected.

By the induction hypothesis, infinitely many simple homogeneous $A^{\prime}$-modules belong to $\mathscr{F}_{*}\left(T^{\prime}\right)=\mathscr{F}_{*}(T)$. Since $\bmod A$ contains only finitely many non-isomorphic selfextending bricks which are not simple homogeneous, we infer that infinitely many simple homogeneous $A$-modules belong to $\mathscr{F}_{*}(T)$, thus establishing our claim.

A dual argument, using [4, (5.2)] and [14, (3.1)], shows that, if $\mathscr{T}_{*}(T)$ is infinite, then infinitely many simple homogeneous $A$-modules belong to $\mathscr{T}_{*}(T)$.

Assume now that $T_{A}$ is a tilting module such that both $\mathscr{T}_{*}(T)$ and $\mathscr{F}_{*}(T)$ are infinite. The above reasoning implies that infinitely many non-isomorphic simple homogeneous $A$-modules belong to each of these subcategories. Let $M \in \mathscr{F}_{*}(T)$ and $N \in \mathscr{T}_{*}(T)$ be two simple homogeneous $A$-modules. It follows from [4, (2.5)] that the supports of $M$ and $N$ are equal, and actually equal to the unique tame concealed full convex subcategory of $A$. Consequently, $\operatorname{dim} M=\operatorname{dim} N$. On the other hand, if $\langle-,-\rangle$ denotes the (homological) bilinear form of $A$ (see $[\mathbf{1 0},(1.5)]$ or $[\mathbf{2},(2.5)]$ ), then

$$
\langle\operatorname{dim} T, \operatorname{dim} M\rangle=-\operatorname{dim}_{k} \operatorname{Ext}_{A}^{1}(T, M)<0
$$

because $\operatorname{Ext}_{A}^{2}(T,-)=0, \operatorname{Hom}_{A}(T, M)=0$ and $\operatorname{Ext}_{A}^{1}(T, M) \neq 0$. Similarly

$$
\langle\operatorname{dim} T, \operatorname{dim} N\rangle>0,
$$

a contradiction which completes the proof of the theorem. 
1.5. Corollary. Let $A$ be a tilted algebra of euclidean type, and $T_{A}$ a tilting (respectively, cotilting) module. Then $\mathscr{T}_{*}(T)$ or $\mathscr{F}_{*}(T)$ (respectively, $\mathscr{T}^{*}(T)$ or $\mathscr{F}^{*}(T)$ ) is finite.

1.6. We end this section by considering another important class of tame algebras, namely that of the tubular algebras $[15,(5)]$. Such an algebra also has a unique preprojective and a unique preinjective component, all other components being called regular. The above results are not true for tubular algebras, as they have regular tilting modules, and regular cotilting modules, which induce infinite torsion and torsion-free classes.

Lemma. Let $A$ be a tubular algebra. Then $A$ has a regular tilting module and a regular cotilting module.

Proof. It follows from the definition of tubular algebras that $A$ contains a full convex subcategory $B$ which is a tilted algebra of euclidean type having a complete slice $\mathscr{S}$ in its preinjective component such that $A$ is a branch extension of $B$ by a branch rooted at a regular indecomposable $B$-module. Let $P_{A}$ be the direct sum of the indecomposable projective $A$-modulus which belong to the regular component, and $U_{A}$ denote the slice module of $\mathscr{S}$, considered as an $A$-module. Then modulo some $\tau$-shift $\mathscr{P}, U_{A}$ may be assumed to be a regular $A$-module (which, in the notation of $[15,(5)]$, lies in $\mathscr{P}_{\infty}$ ). The module

$$
T_{A}=P_{A} \oplus \tau_{A}^{-1} U
$$

(where $\tau_{A}$ denotes the Auslander-Reiten translation in $\bmod A$ ) is clearly a regular tilting $A$-module. Observe that, by $[\mathbf{1 5},(5.2)$ (5)], End $T$ is again a tubular algebra. On the other hand, both $\mathscr{T}_{*}(T)$ and $\mathscr{F}_{*}(T)$ are infinite, as they respectively contain all the preinjective and all the preprojective $A$-modules.

The statement about cotilting modules follows from the fact that $A^{\text {op }}$ is cotubular and hence, by $[15,(5.2)(3)]$, also tubular.

\section{Tilted algebras.}

2.1. Lemma. Let $A$ be a representation-infinite tilted algebra of euclidean type.

(i) Assume that $A$ has a complete slice $\mathscr{S}$ in its preinjective component, and let $M_{A}$ be a predecessor of $\mathscr{S}$ such that $\operatorname{Ext}_{A}^{1}(M, M)=0$. Then the class $\mathscr{F}_{*}(M)=$ $\left\{X_{A} \mid \operatorname{Hom}_{A}(M, X)=0\right\}$ or the class $\operatorname{Gen}\left(M_{A}\right)$ of all $A$-modules generated by $M$ is finite.

(ii) Assume that $A$ has a complete slice $\mathscr{S}$ in its preprojective component, and let $M_{A}$ be a successor of $\mathscr{S}$ such that $\operatorname{Ext}_{A}^{1}(M, M)=0$. Then the class $\mathscr{T}_{*}(M)=$ $\left\{X_{A} \mid \operatorname{Ext}_{A}^{1}(M, X)=0\right\}$ or the class $\operatorname{Cogen}(\tau M)$ of all $A$-modules cogenerated by $\tau M$ is finite.

Proof. We shall first prove (i). It follows from the hypothesis that there exists a tame hereditary algebra $B$, and a tilting module $T_{B}$ without preinjective direct summands such that $A=$ End $T_{B}$ and $\mathscr{Y}_{*}(T)$ consists of the predecessors of $\mathscr{S}$.

Assume first that $M$ has an indecomposable preprojective direct summand $M_{0}$. We claim that in this case $\mathscr{F}_{*}(M)$ is finite. Indeed, since $\mathscr{F}_{*}(M) \subseteq \mathscr{F}_{*}\left(M_{0}\right)$ and $\mathscr{X}_{*}(T)$ is finite, the finiteness of $\mathscr{F}_{*}(M)$ would follow from the finiteness of the class

$$
\mathscr{F}_{*}\left(M_{0}\right) \cap \mathscr{Y}_{*}(T)=\left\{X_{A} \in \mathscr{Y}_{*}(T) \mid \operatorname{Hom}_{A}\left(M_{0}, X\right)=0\right\}
$$


Now, the image of any $X \in \mathscr{F}_{*}\left(M_{0}\right) \cap \mathscr{Y}_{*}(T)$ under the equivalence $-\bigotimes_{A} T: \mathscr{Y}_{*}(T) \Im$ $\mathscr{T}_{*}(T)$ belongs to the class

$$
\mathscr{F}_{*}\left(M_{0} \otimes T\right)=\left\{Y_{B} \mid \operatorname{Hom}_{B}\left(M_{0} \otimes_{A} T, Y\right)=0\right\}
$$

that is, $\left[\mathscr{F}_{*}\left(M_{0}\right) \cap \mathscr{Y}_{*}(T)\right] \otimes_{A} T \subseteq \mathscr{F}_{*}\left(M_{0} \otimes_{A} T\right)$. However the latter class is finite, since $M_{0} \otimes_{A} T$ is an indecomposable preprojective $B$-module and $B$ is tame hereditary. This completes the proof of our claim.

Next, assume that $M$ has no preprojective direct summand. We shall show that in this case $\operatorname{Gen}\left(M_{A}\right)$ is finite. Again, the finiteness of $\operatorname{Gen}\left(M_{A}\right)$ would follow from the finiteness of the class

$$
\operatorname{Gen}(M) \cap \mathscr{Y}_{*}(T)=\left\{X_{A} \in \mathscr{Y}_{*}(T) \mid X \text { is generated by } M\right\} \text {. }
$$

Now, the image of any $X \in \operatorname{Gen}(M) \cap \mathscr{Y}_{*}(T)$ under the right exact functor $-\bigotimes_{A} T$ clearly belongs to the class

$$
\operatorname{Gen}\left(M \otimes_{A} T\right)=\left\{Y_{B} \mid Y \text { is generated by } M \otimes_{A} T\right\}
$$

that is, $\left[\operatorname{Gen}(M) \cup \mathscr{Y}_{*}(T)\right] \otimes_{A} T \subseteq \operatorname{Gen}\left(M \otimes_{A} T\right)$. Applying the construction dual to that of Bongartz in $[7,(2.1)]$, let

$$
0 \rightarrow\left(M \otimes_{A} T\right)^{d} \rightarrow E_{B} \rightarrow(D M)_{B} \rightarrow 0
$$

be the universal exact sequence, where $d=\operatorname{dim}_{k} \operatorname{Ext}_{B}^{1}\left(D B, M \otimes_{A} T\right)$. It follows from [7, (2.1)] that $U_{B}=\left(M \otimes_{A} T\right) \oplus E$ is a tilting module. Since, as observed in [15, (4.1)(1)], the indecomposable summands of $E$ are injective or successors of $M \otimes_{A} T$, then $U$ has no preprojective direct summand. By $[11,(3.2)], \mathscr{T}_{*}(U)=\operatorname{Gen}(U)$ is finite. Consequently so is $\operatorname{Gen}(M) \cap \mathscr{Y}_{*}(T)$. This completes the proof of (i).

The proof of (ii) is dual: if $M$ has a preinjective direct summand, we prove as above that $\mathscr{T}_{*}(M)$ is finite, while if $M$ has no preinjective direct summand, the finiteness of Cogen $(\tau M)$ is proved using the fact that, if $T_{B}$ is a tilting module over a hereditary algebra $B$ such that End $T_{B}=A$, then the functor $\operatorname{Tor}_{1}^{A}(-, T)$ is left exact, because $\operatorname{Ext}_{A}^{2}(-, T)=0$.

Remark. Under the stated hypothesis, $M$ is a partial tilting module. The above lemma may then be used to give an alternative proof of (1.5).

2.2. Recall that an algebra $A$ is called a finite enlargement of a full convex subcategory $B$ if all but at most finitely many non-isomorphic indecomposable $A$-modules have their support entirely contained in $B$.

Corollary. Let $A$ be a finite enlargement in the preinjective (respectively, preprojective) component of a tilted algebra $B$ of euclidean type having a complete slice in that component. Then, for every tilting module $T_{A}, \mathscr{T}_{*}(T)$ or $\mathscr{F}_{*}(T)$ is finite.

Proof. We shall assume that $A$ is a finite enlargement in the preinjective component of $B$ (the other case is treated similarly). Let $\mathscr{S}$ be a complete slice in the preinjective component such that all predecessors of $\mathscr{S}$ are $B$-modules. If $T$ is a successor of $\mathscr{S}$, clearly $\mathscr{T}_{*}(T)$ is finite. Otherwise, let $T_{0}$ denote the direct sum of all indecomposable summands of $T$ which precede $\mathscr{S}$. By $(2.1), \mathscr{F}_{*}\left(T_{0}\right)=\left\{X_{B} \mid \operatorname{Hom}_{B}\left(T_{0}, X\right)=0\right\}$ or $\operatorname{Gen}\left(T_{0}\right)=\left\{X_{B} \mid X\right.$ is generated by $\left.T_{0}\right\}$ is finite. Since $A$ is a finite enlargement of $B$, also $\mathscr{T}_{*}(T)$ or $\mathscr{F}_{*}(T)$ is finite. 
2.3. For tilted algebras of wild type, we shall use the notations and results of [14]. In particular, let $\Delta$ be a wild quiver, $B=k \Delta$ and $T=\bigoplus_{i=1}^{n} T_{i}$ a tilting $B$-module such that $A=$ End $T_{B}$ (where $T_{i}$ is indecomposable for all $i$ ). We let $I$ (respectively, $J$ ) denote the set of all $i(1 \leq i \leq n)$ such that $\operatorname{Ext}_{A}^{1}\left(T_{i}, M\right) \neq 0$ (respectively, $\operatorname{Hom}_{A}\left(T_{i}, M\right) \neq 0$ ) for only finitely many indecomposables $M \in \mathscr{F}_{*}(T)$ (respectively, $M \in \mathscr{T}_{*}(T)$ ). The algebras $A_{\infty}=\operatorname{End}\left(\bigoplus_{i \in l}, T_{i}\right)$ and ${ }_{\infty} A=\operatorname{End}\left(\bigoplus_{i \in J} T_{i}\right)$ are respectively called the right end algebra and the left end algebra of $A$. Then

(i) $A$ is an iterated one-point extension of $\infty_{\infty} A$, and

(ii) there is a hereditary quotient algebra ${ }_{\infty} B$ of $B$ and a tilting ${ }_{\infty} B$-module ${ }_{\infty} T^{\prime}$ without preinjective direct summands such that $\operatorname{End}\left({ }_{\infty} T^{\prime}\right)={ }_{\infty} A$. Moreover, ${ }_{\infty} B=B_{1} \times \ldots \times B_{t}$, with $B_{i}$ connected for all $i,{ }_{\infty} T^{\prime}=T_{1}^{\prime} \oplus \ldots \oplus T_{t}^{\prime}$, where $T_{i}^{\prime}$ is a tilting $B_{i}$-module without preinjective direct summands and ${ }_{\infty} A=A_{1} \times \ldots \times A_{t}$ for $A_{i}=$ End $T_{i}^{\prime}$.

The dual statements holds for $A_{\infty}$.

The Auslander-Reiten quiver $\Gamma_{A}$ has the following shape.

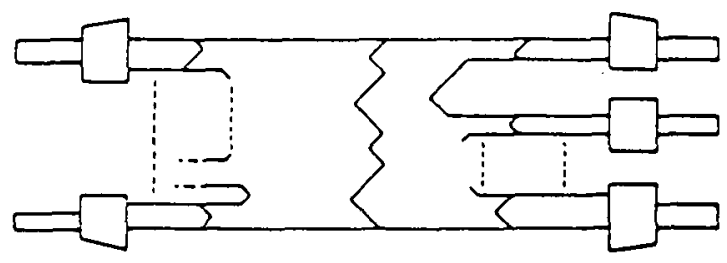

Again, it has preprojective and preinjective components, all the other being called regular. A component containing a complete slice is called a connecting component.

2.4. Proof of Theorem B. We shall first show that (i) implies (ii). The statement is trivial if $A$ is representation-finite, and follows from (1.5) if $A$ is of euclidean type. We may thus assume that $A$ is a representation-infinite tilted algebra of wild type.

First, one of the end algebras ${ }_{\infty} A$ or $A_{\infty}$ is zero. Indeed, if this is not the case, the slice module $T_{A}$ of a complete slice in the connecting component is a tilting (and also a cotilting) module with both $\mathscr{T}_{*}(T)$ and $\mathscr{F}_{*}(T)$ (and also $\mathscr{T}^{*}(T)$ and $\mathscr{F}^{*}(T)$ ) infinite.

Without loss of generality, we may supppose that $A_{\infty}$ is zero. We claim that ${ }_{\infty} A$ is connected. Indeed, if this is not the case, then we can write ${ }_{\infty} A=A_{1} \times A_{2}$ with $A_{1}$ connected, and both $A_{1}$ and $A_{2}$ representation-infinite. By (2.3)(ii), $A_{1}$ is a tilted algebra having a complete slice $\mathscr{S}$ in the preinjective component, and $\mathscr{S}$ may be chosen so that any predecessor of $\mathscr{S}$ in $\bmod A$ is an $A_{1}$-module. Let $T_{1}$ be the slice module of $\mathscr{S}$, considered as an $A$-module, let $e_{1}$ denote the identity of $A_{1}$, and 1 the identity of $A$; then $T=T_{1} \oplus\left(1-e_{1}\right) A$ is a tilting $A$-module. Since all indecomposable regular $A_{1}$-modules belong to $\mathscr{F}_{*}(T)$, then $\mathscr{F}_{*}(T)$ is infinite, and since all $A_{2}$-modules belong to $\mathscr{T}_{*}(T)$, it is infinite as well. This shows that ${ }_{\infty} A$ is connected.

We shall now show that ${ }_{\infty} A$ is tame or has only two non-isomorphic simple modules (it is then necessarily hereditary). In order to prove it, we shall assume that this is not the case and construct a tilting module $T_{A}$ such that both $\mathscr{T}_{*}(T)$ and $\mathscr{F}_{*}(T)$ are infinite. Now, there exists a wild hereditary algebra $B$ and a tilting module $U_{B}$ such that ${ }_{\infty} A=$ End $U_{B}$. Then $U_{B}$ has no preinjective direct summands, and $\mathscr{Y}_{*}(U)$ is a cofinite full subcategory of the category $\bmod A$, closed under predecessors. By $[14,(2.1)]$, each regular component $\mathscr{C}$ 
of $\Gamma_{B}$ contains a complete cone $\Gamma_{\mathscr{C}}$, closed under predecessors and entirely contained in $\mathscr{T}_{*}(U)$. By $[16,6], B$ has a regular tilting module $V$. We clearly may assume that all the summands of $V$ belong to the cones $\Gamma_{\mathscr{C}}$. Consequently, $T_{0}=\operatorname{Hom}_{B}(U, V)$ is a regular partial tilting $A$-module. Let $P_{A}$ denote the direct sum of all non-isomorphic indecomposable projectives which belong to the preinjective component of $\Gamma_{A}$. Then the module $T_{A}=T_{0} \oplus P$ satisfies our assertion.

If ${ }_{\infty} A$ is zero, the proof is entirely analogous using cotilting modules.

We shall finally prove that, conversely, (ii) implies (i). If $A$ is tame and one-parametric then, by (2.3) or [14, (4.2b)], $A$ is a finite enlargement of a tilted algebra of euclidean type and the statement follows from (2.2). If $A$ is wild, one of its end algebras is zero and the other is hereditary with two simple modules. By [13, (2.6)], all regular indecomposable modules have self-extensions and consequently all the summands of a tilting (or cotilting module are preprojective or preinjective. Assume that $A_{\infty}$ is zero. If a tilting module $T_{A}$ has a preprojective summand $T_{0}$, then $\mathscr{F}_{*}(T) \subseteq \mathscr{F}_{*}\left(T_{0}\right)=\left\{M_{A} \mid \operatorname{Hom}_{A}\left(T_{0}, M\right)=0\right\}$, which consists of predecessors of $T_{0}$ (and $T_{0}$ has only finitely many non-isomorphic predecessors) and possibly modules whose support is not contained in ${ }_{\infty} A$ (and $A$ is a finite enlargement of ${ }_{\infty} A$ ). If $T_{A}$ has no preprojective summand, it is preinjective, $\mathscr{T}_{*}(T)=\operatorname{Gen}(T)$ consists then of successors of $T$, and $T$ has only finitely many non-isomorphic successors. The proofs of the cases where ${ }_{\infty} A$ is zero, or where $T$ is a cotilting module are dual.

2.5. EXAMPLE. Let $A$ be the algebra given by the quiver

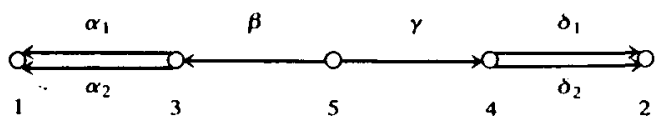

bound by $\beta \alpha_{1}=0, \beta \alpha_{2}=0, \gamma \delta_{1}=0, \gamma \delta_{2}=0$. Then $A$ is a tilted algebra having a complete slice in its preinjective component and ${ }_{\infty} A$ is the direct product of two copies of the Kronecker algebra. The Auslander-Reiten quiver $\Gamma_{A}$ is given by

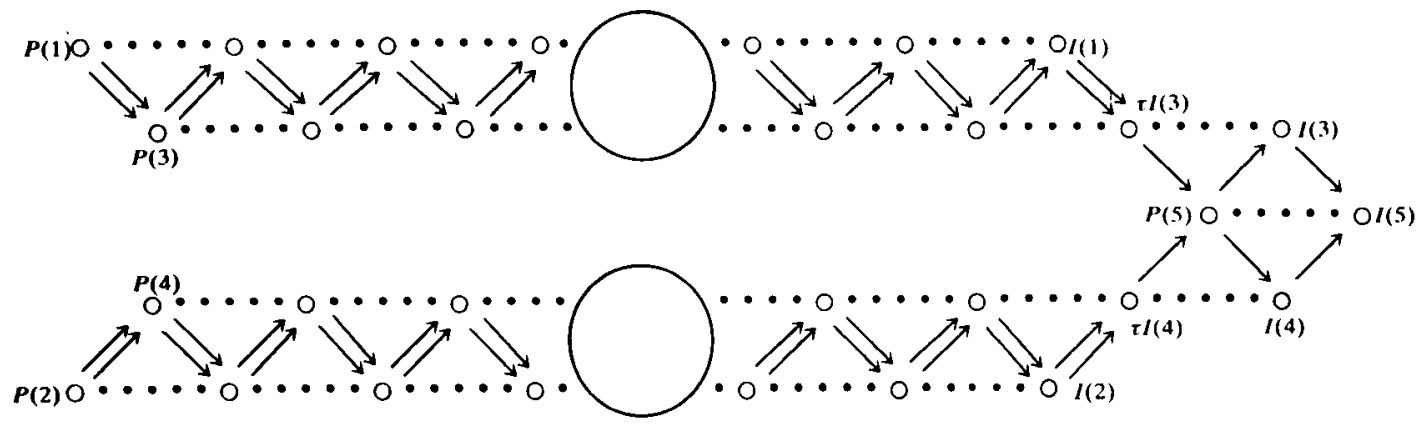

The indecomposable $A$-modules with injective dimension at most one are precisely $I(1)$, $I(2), \tau I(3), \tau I(4), I(3), I(4), I(5)$ and $P(5)$. Any cotilting module $T_{A}$ must then have these as summands and hence $\mathscr{T}^{*}(T)$ is always finite. On the other hand,

$$
T_{A}^{\prime}=P(2) \oplus P(4) \oplus P(5) \oplus I(1) \oplus \tau I(3)
$$

is a tilting module with both $\mathscr{T}_{*}\left(T^{\prime}\right)$ and $\mathscr{F}_{*}\left(T^{\prime}\right)$ infinite.

ACKNOWLEDGEMENTS. This paper was written while the second author was visiting the first at the Université de Sherbrooke. Both gratefully acknowledge support from the Natural Sciences and Engineering Research Council of Canada. 


\section{REFERENCES} 899-913.

1. I. Assem, Torsion theories induced by tilting modules, Canad. J. Math. 36 (1984),

2. I. Assem, Tilting theory-an introduction. Topics in algebra, Rings and representations of algebras, Banach Center Publ. 26 part 1 (PWN, 1990), 127-180.

3. I. Assem and A. Skowronski, Algebras with cycle-finite derived categories, Math. Ann. 280 (1988), 441-463.

4. I. Assem and A. Skowronski, Quadratic forms and iterated tilted algebras, J. Algebra 128 (1990), 55-85.

5. M. Auslander and I. Reiten, Representation theory of Artin algebras. III. Almost split sequences, and IV. Invariants given by almost split sequences, Comm. Algebra 3 (1975), 239-294, and 5 (1977), 443-518. 751-757.

6. D. Baer, A note on wild quiver algebras and tilting modules, Comm. Algebra 17 (1989),

7. K. Bongartz, Tilted algebras, Representations of algebras (Puebla, 1980), Lecture Notes in Math. 903 (Springer, 1981), 26-38.

8. K. Bongartz and P. Gabriel, Covering spaces in representation-theory, Invent. Math. 65 $(1981 / 82), 331-378$.

9. D. Happel, Triangulated categories in the representation theory of finite-dimensional algebras, London Mathematical Society Lecture Note Series 119 (Cambridge University Press, 1988).

10. D. Happel and C. M. Ringel, Tilted algebras, Trans. Amer. Math. Soc. 274 (1982), 399-443.

11. D. Happel and C. M. Ringel, Construction of tilted algebras, Representations of algebras (Puebla, 1980), Lecture Notes in Math. 903 (Springer, 1981), 125-144.

12. M. Hoshino, Tilting modules and torsion theories, Bull. London Math. Soc. 14 (1982), 334-336.

13. M. Hoshino, Modules without self-extensions and Nakayama's conjecture, Arch. Math. (Basel) 43 (1984), 493-500.

14. O. Kerner, Tilting wild algebras, J. London Math. Soc. (2) 39 (1989), 29-47.

15. C. M. Ringel, Tame algebras and integral quadratic forms, Lecture Notes in Math. 1099 (Springer, 1984).

16. C. M. Ringel, The regular components of the Auslander-Reiten quiver of a tilted algebra, Chinese Ann. Math. Ser. B 9 (1988), 1-18.

17. A. Skowronski, Algebras of polynomial growth, Topics in algebra, Rings and representations of algebras, Banach Center Publ. 26 part 1 (PWN, 1990), 535-568.

18. S. O. Smalø, Torsion theories and tilting modules, Bull. London Math. Soc. 16 (1984), $518-522$.

IBRAHIM ASSEM

MathÉMATIQUES ET INFORMATIQUE

UNIVERSITÉ de SHERBRoOKE

SHERBROOKE, QUÉBEC

CANADA, J1K 2R1

OTtO KeRner

Mathematisches INSTITUT

HeINRICH HeINE UniversitäT

UNIVERSITÄTSTR. 1

D-4000 DÜSSELDORF 1

Federal Republic of Germany 